\section{Diagnosing ectopic pregnancy in the emergency setting}

\author{
Robert Lee, Carolyn Dupuis, Byron Chen, Andrew Smith, Young H. Kim \\ Department of Radiology, University of Massachusetts Medical School, Worcester, MA, USA
}

Ectopic pregnancy is the implantation of a fertilized egg outside the uterine endometrial cavity. For women presenting to the emergency department with abdominal pain and/or vaginal bleeding, ectopic pregnancy is an important diagnostic consideration. The diagnosis is made based on laboratory values and ultrasound imaging findings. The ultrasound appearance of both normal early pregnancy and ectopic pregnancy are variable and often subtle, presenting diagnostic challenges for radiologists. This pictorial essay describes and illustrates the sonographic findings of ectopic pregnancy and reviews the differential diagnoses that can mimic ectopic pregnancy on ultrasound. With the possibility of medical management, the value of early detection and prompt initiation of treatment has increased in improving clinical outcomes and preventing the complications of ectopic pregnancy.

Keywords: Pregnancy, ectopic; Ultrasonography; Diagnosis, differential; Methotrexate

\section{Introduction}

Ectopic pregnancy is the implantation of a fertilized egg outside the endometrial cavity of the uterus. In women who present to the emergency department (ED) complaining of symptoms of abdominal/pelvic pain or vaginal bleeding, and are pregnant, ectopic pregnancy is a major diagnostic consideration.

\section{Epidemiology}

In the early 1990s, the Centers for Disease Control estimated the rate of ectopic pregnancy to be about $2 \%$ of all pregnancies [1]. In pregnant women who presented to the ED with abdominal pain and/or vaginal bleeding, the incidence was found to be significantly higher, ranging from $6 \%$ to $16 \%$ [2].

A major risk factor for ectopic pregnancy is pelvic inflammatory disease [3], and other high-risk factors include a previous ectopic pregnancy and tubal surgery. Moderate to low added risk is seen with intrauterine contraceptive devices, multiple sexual partners, smoking, and assisted reproductive technology, including in vitro fertilization (IVF).

Approximately $98 \%$ of ectopic pregnancies occur in the fallopian tubes. Among those, $70 \%$ of tubal ectopic pregnancies occur within the ampullary portion, followed by the isthmus, fimbriae, and interstitial tubal segments [4]. The remaining ectopic pregnancies can be found in various locations outside of the fallopian tubes, including the ovary, cesarean section scars, cervix, and peritoneal cavity.

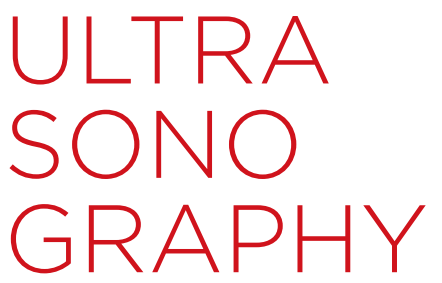

PICTORIAL ESSAY

https://doi.org/10.14366/usg. 17044 pISSN: 2288-5919 - elSSN: 2288-5943 Ultrasonography 2018;37:78-87

Received: June 21, 2017 Revised: August 14, 2017 Accepted: August 19, 2017

Correspondence to: Young H. Kim, MD, Department of Radiology, University of Massachusetts Medical School, 55 N Lake Ave, Worcester, MA 01655, USA

Tel. +1-508-334-7237

Fax. +1-508-856-1860 E-mail: Young.Kim@umassmemorial. org

This is an Open Access article distributed under the terms of the Creative Commons Attribution NonCommercial License (http://creativecommons.org/ licenses/by-nc/3.0/) which permits unrestricted noncommercial use, distribution, and reproduction in any medium, provided the original work is properly cited.

Copyright @ 2018 Korean Society of Ultrasound in Medicine (KSUM)

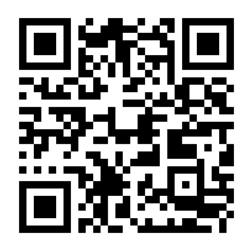

How to cite this article:

Lee R, Dupuis C, Chen B, Smith A, Kim YH. Diagnosing ectopic pregnancy in the emergency setting. Ultrasonography. 2018 Jan;37(1):78-87. 


\section{Imaging Technique}

Ultrasound (US) is the imaging modality of choice for symptomatic women in early pregnancy presenting to the ED. Ideally, pelvic US for these patients includes both transabdominal and transvaginal evaluation. Transabdominal evaluation (Fig. 1A, B) is performed with a low-middle frequency transducer $(1-5 \mathrm{MHz})$ after adequate filling of the bladder. It includes a wider field of view of the pelvis and provides better visualization of the uterine fundus and superiorly positioned adnexa. It can also be used to visualize free fluid or hemorrhage within the peritoneal cavity.

A transvaginal examination (Fig. 2A, B) is performed with a high- frequency intravaginal probe $(>7 \mathrm{MHz})$ after voiding. It provides superior near-field resolution, allowing for a detailed evaluation of the endometrial cavity. Transvaginal US also affords a more detailed assessment of the ovaries and other adnexal structures. This is of the utmost importance in patients without an identifiable intrauterine pregnancy (IUP).

\section{Clinical and Diagnostic Approach}

For any premenopausal woman presenting to the ED with abdominal pain and/or vaginal bleeding, a pregnancy test should be conducted. Serum $\beta$-human chorionic gonadotropin ( $\beta$-hCG) is a more sensitive

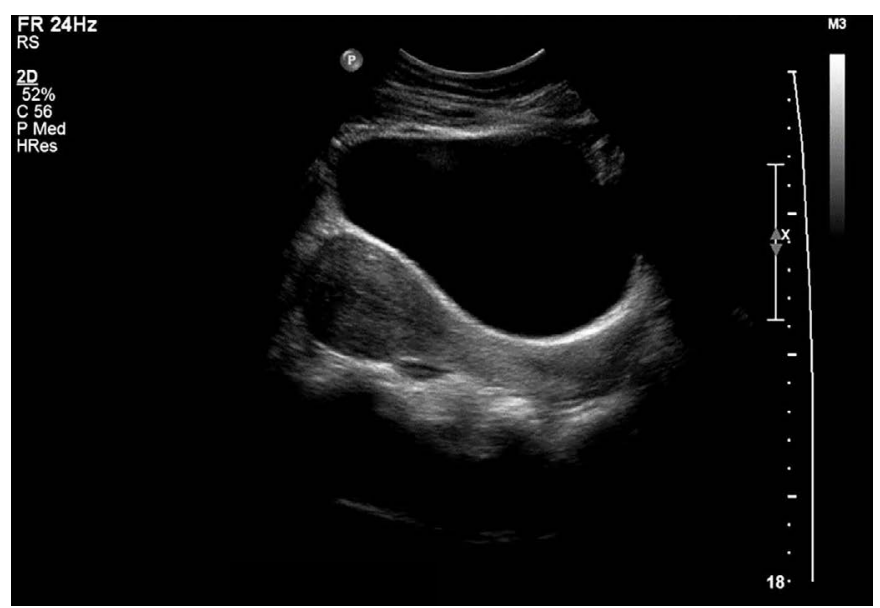

A

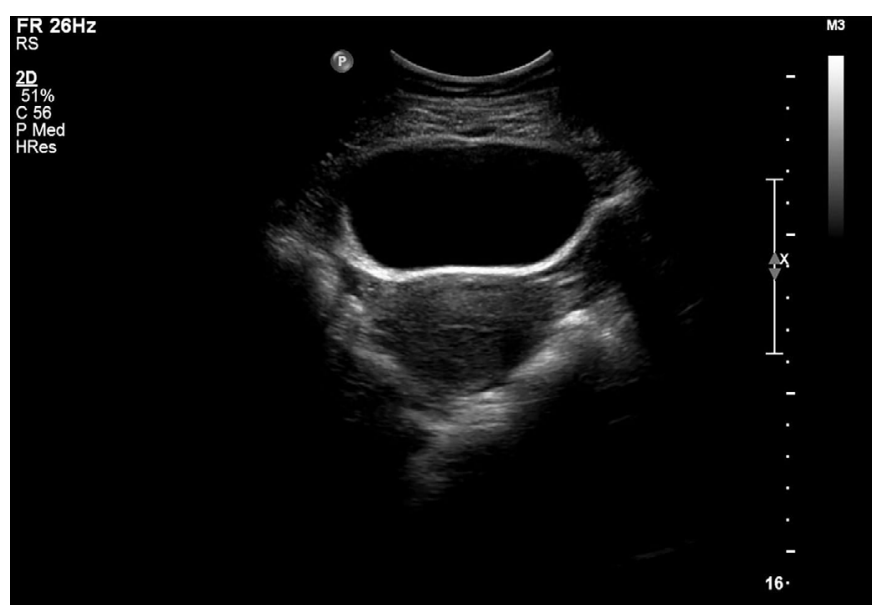

B

Fig. 1. Transabdominal views of the pelvis.

A, B. Transabdominal sagittal and transverse views of the pelvis demonstrate a normal uterus and urinary bladder.

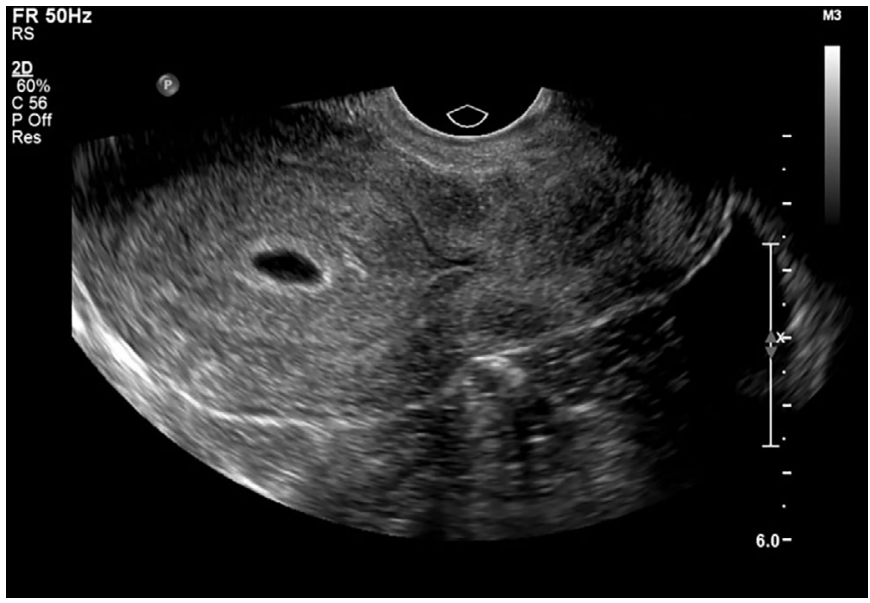

A

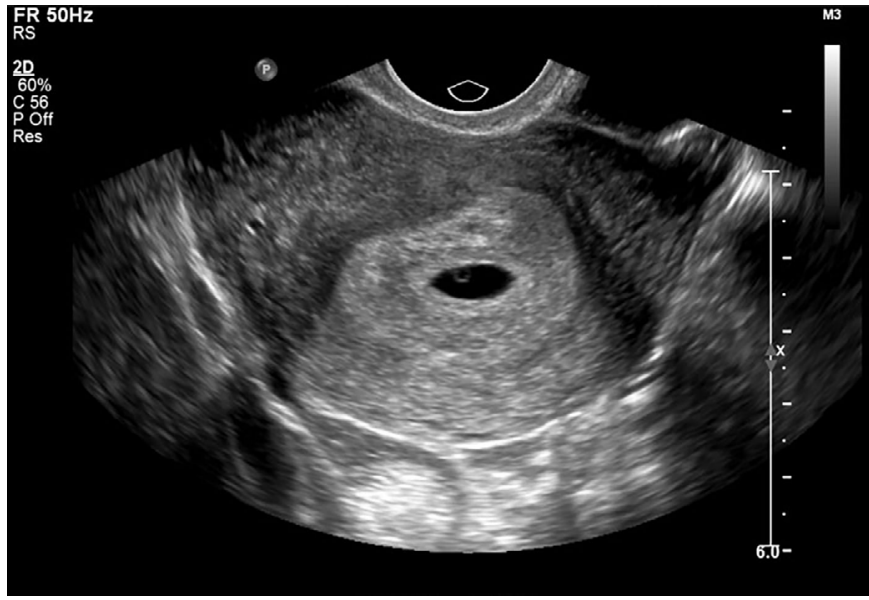

B

Fig. 2. Transvaginal views of the pelvis.

A, B. Transvaginal sagittal and transverse views allow better visualization of the endometrium. An intrauterine gestational sac is seen with a yolk sac. No embryo is yet visualized in this patient with an early pregnancy, at approximately 5-6 weeks. 
test than urinary hCG for confirming pregnancy, and negative serum $\beta$-hCG essentially excludes a live pregnancy [5]. Patients with a positive pregnancy test and symptoms suggestive of an ectopic pregnancy are then evaluated with pelvic US to determine the presence or absence of an IUP and to examine potential locations of the ectopic pregnancy (Fig. 3).

The sonographic visualization of an intrauterine gestational sac containing a yolk sac and/or embryo (Fig. 2A, B) along with normal adnexal structures essentially excludes the possibility of an ectopic pregnancy [6].

If no IUP is identified on US, the sonographic findings should be carefully interpreted in the context of the patient's clinical information, particularly the estimated gestational age according to the last menstrual period and the serum $\beta$-hCG level relative to the discriminatory zone. The discriminatory zone is the serum $\beta$-hCG level above which an IUP is expected to be visualized with US. For transvaginal US, the discriminatory zone ranges from 1,500 to 2,000 $\mathrm{mIU} / \mathrm{mL}$ at most institutions [7]. The discriminatory zone is higher for transabdominal US, at 6,000-6,500 $\mathrm{mlU} / \mathrm{mL}$ [8]. A patient with $\beta$-hCG levels below the discriminatory zone, in whom no IUP is visualized and has normal-appearing adnexa, may simply have a very early normal pregnancy below the detection threshold for US. In such cases, clinical and US follow-up are recommended. Serial serum $\beta$-hCG levels should be obtained to monitor the appropriate increase in the values as the pregnancy progresses.

When there is no sonographic evidence of IUP in patients with a $\beta$-hCG level above the discriminatory zone, an alternative source for the $\beta$-hCG must be considered. The bilateral adnexae must be carefully scrutinized, as ectopic pregnancy can have variable, and often subtle, sonographic findings. Even in such patients with apparently normal bilateral adnexae, ectopic pregnancy should be included as a part of the differential diagnosis. It is estimated that $15 \%-35 \%$ of patients with ectopic pregnancy do not have an extrauterine mass identified on transvaginal US [5]. These patients must be closely followed with repeat US and serial $\beta$-hCG tests.

\section{US Findings of Tubal Ectopic Pregnancy}

Tubal ectopic pregnancy most commonly manifests as an extraovarian heterogeneous mass, usually representative of a

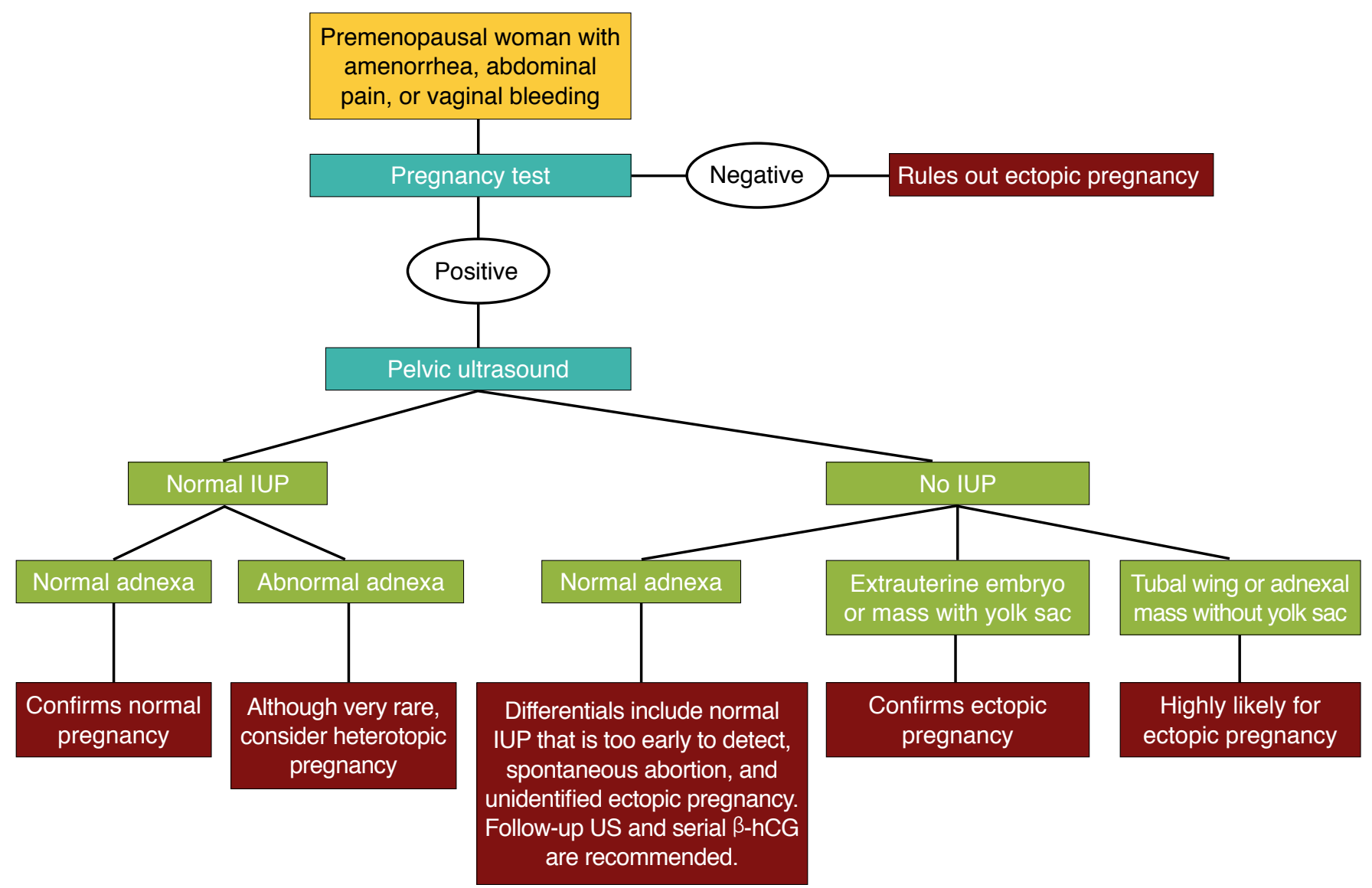

Fig. 3. Diagnostic algorithm for ectopic pregnancy. IUP, intrauterine pregnancy; US, ultrasonography; $\beta$-hCG, $\beta$-human chorionic gonadotropin. 
hematoma at the site of ectopic implantation (Fig. 4). The mass may demonstrate various degrees of echogenicity depending on the age of the blood products.

Tubal ectopic pregnancy can be also seen as an echogenic ring in the adnexa surrounding an unruptured ectopic pregnancy, which is known as the tubal ring sign (Fig. 5A). This is the second most common sign of ectopic pregnancy and has a 95\%

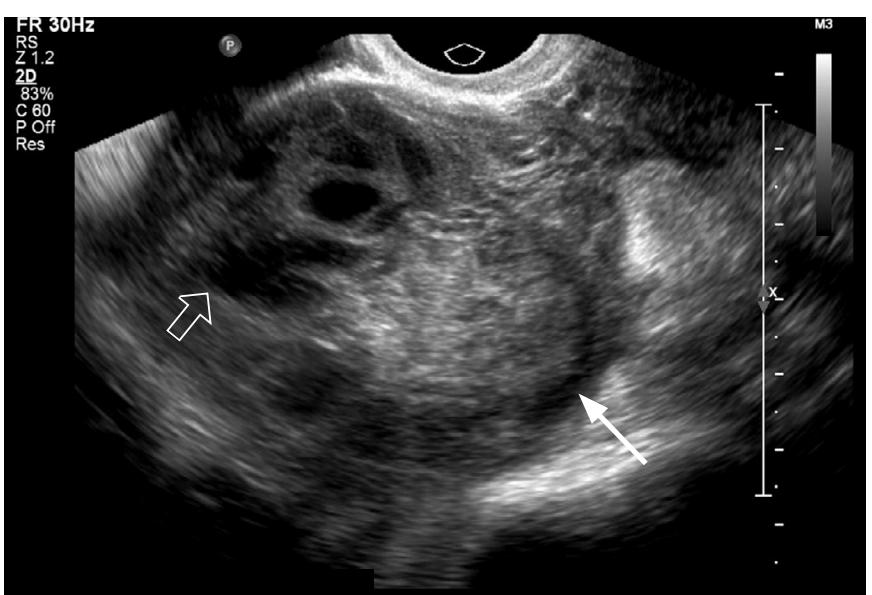

Fig. 4. Ectopic pregnancy seen as a hematoma. In this 21-year-old woman with positive serum pregnancy test and vaginal bleeding, a complex echogenic mass (arrow) is seen in the right adnexa, which separates from the right ovary (open arrow) with applied pressure during transvaginal ultrasound. The echogenic adnexal mass is representative of a hematoma at the site of ectopic implantation. The patient was treated surgically.

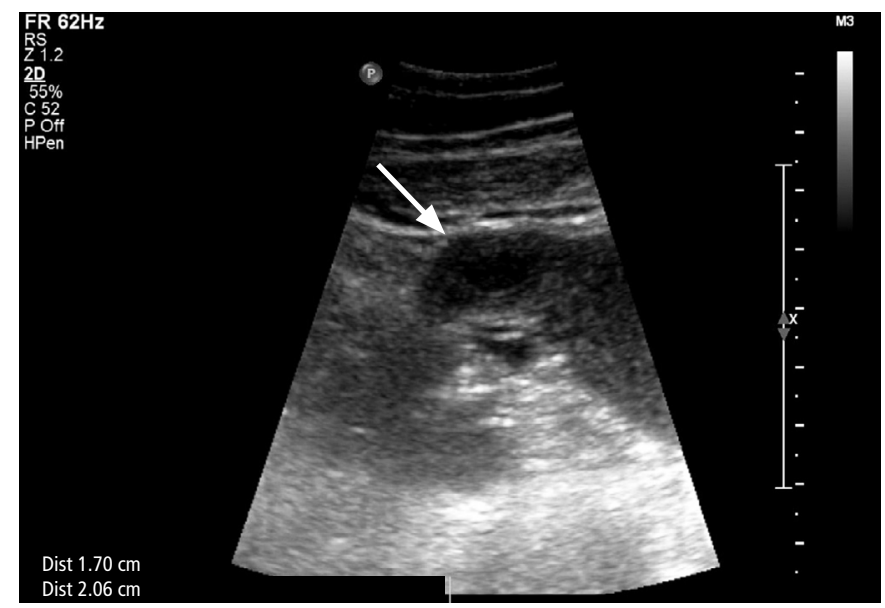

A

Fig. 5. Tubal ring sign.

A. In this 20-year-old woman with a positive pregnancy test presenting to the emergency department with pelvic pain and vaginal spotting, there is an adnexal mass with echogenic ring (arrow). B. A color Doppler image of the right adnexa shows increased vascularity in the echogenic ring. The patient was diagnosed with ectopic pregnancy based on a clinical and sonographic assessment and was treated successfully with methotrexate.

positive predictive value [9]. Color Doppler often shows a ring of circumferential vascularity (Fig. 5B).

Visualization of an extrauterine gestational sac with an embryo (Fig. 6A, B) directly confirms an ectopic pregnancy, but this finding is rarely observed [4].

Additional sonographic findings are associated with ectopic pregnancy. The presence of free intraperitoneal fluid (Fig. 7) in patients with a positive pregnancy test and empty uterus has $69 \%$ specificity and $63 \%$ sensitivity for ectopic pregnancy [10]. While free fluid is a nonspecific finding that can be seen both physiologically and in other pathologies, a moderate or large volume of free fluid greater than expected for a physiologic volume and complexity of the free fluid, with floating debris, blood products, and/or organized blood clots (Fig. 8) are suspicious features for ectopic pregnancy.

A so-called pseudogestational sac represents a small amount of intrauterine fluid (Fig. 9A), which can be misinterpreted as a true gestational sac in cases of ectopic pregnancy (Fig. 9B). It is estimated to be seen in $10 \%$ of patients with ectopic pregnancies [11]. Real-time observation of this phenomenon will often show a shifting of the fluid as the exam progresses, unlike the fixed position of a true intrauterine gestational sac.

\section{Other Rare Ectopic Pregnancies}

In an interstitial ectopic pregnancy, the implantation takes place in the interstitial portion of the fallopian tube, and this accounts for $2 \%-4 \%$ of ectopic pregnancies [11]. Interstitial pregnancy can

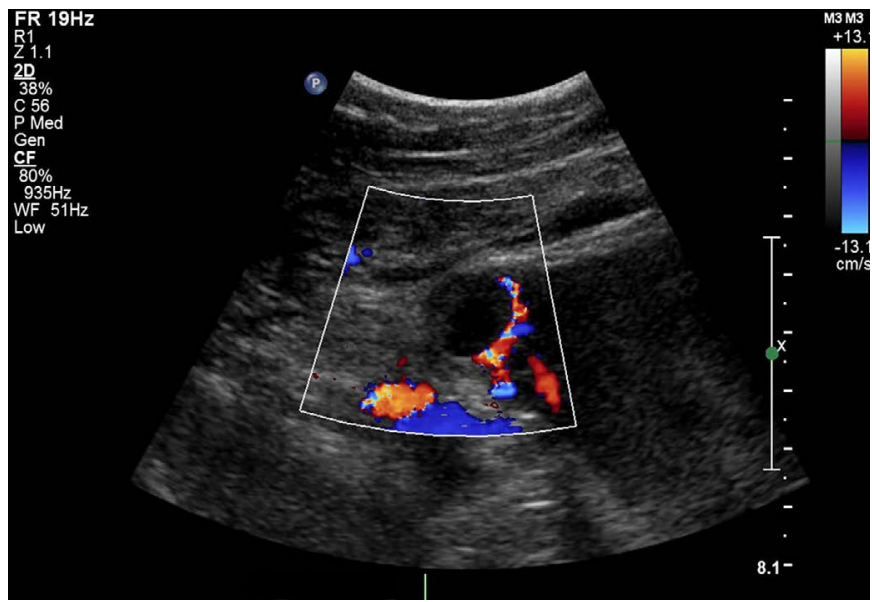

B 
be especially dangerous due to a high risk of profuse hemorrhage resulting from its propensity for delayed rupture at a larger size and its proximity to the intramyometrial arcuate vasculature. It can be difficult to distinguish on US from a normal IUP that is eccentrically positioned. The diagnosis is suggested when a gestational sac is visualized high in the fundus surrounded by a thin layer of myometrium measuring less than $5 \mathrm{~mm}$ [11]. The interstitial line sign is an echogenic line extending from the endometrium to the ectopic gestational sac, and is also helpful in diagnosis [12].

Cesarean section scar ectopic pregnancy accounts for fewer than $1 \%$ of ectopic pregnancies [13]. A gestational sac is found within the myometrium of the anterior lower uterine segment at the site of a prior cesarean section scar. Severe myometrial thinning or complete absence of the myometrium between the gestational sac and the bladder will often be seen. Scar ectopic pregnancies pose a significantly increased risk for uterine rupture.

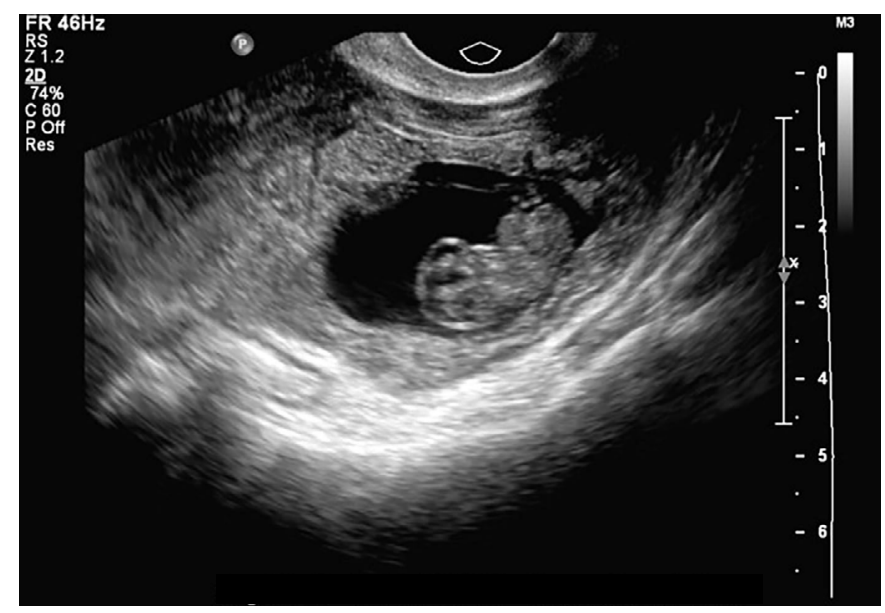

A

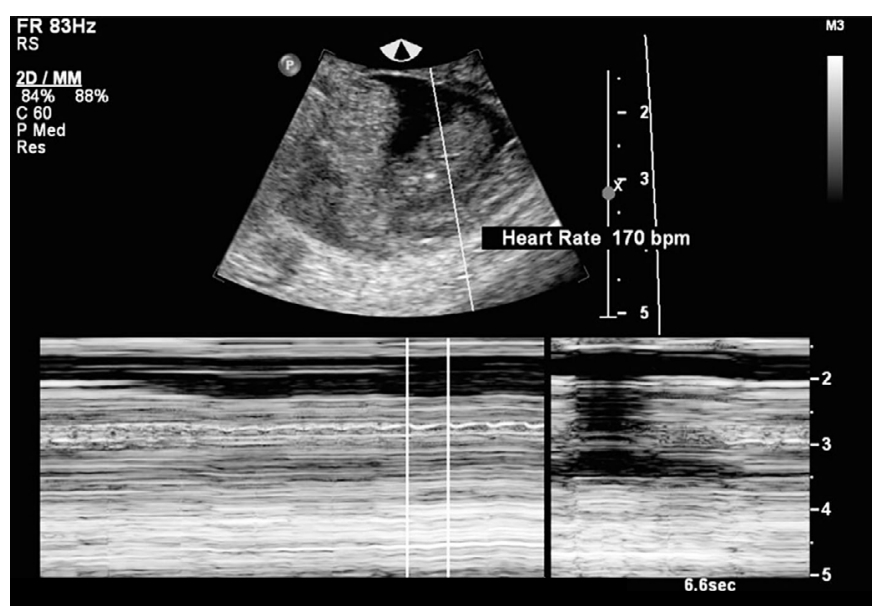

B

Fig. 6. Live tubal ectopic pregnancy.

A. In this 25-year-old woman with a positive pregnancy test and vaginal bleeding, there was a gestational sac containing an embryo in the left adnexa, outside the left ovary. B. The M-mode ultrasound of the embryo in the left adnexa shows a fetal heart rate of 170 beats per minute. These findings are confirmatory of a live tubal ectopic pregnancy.

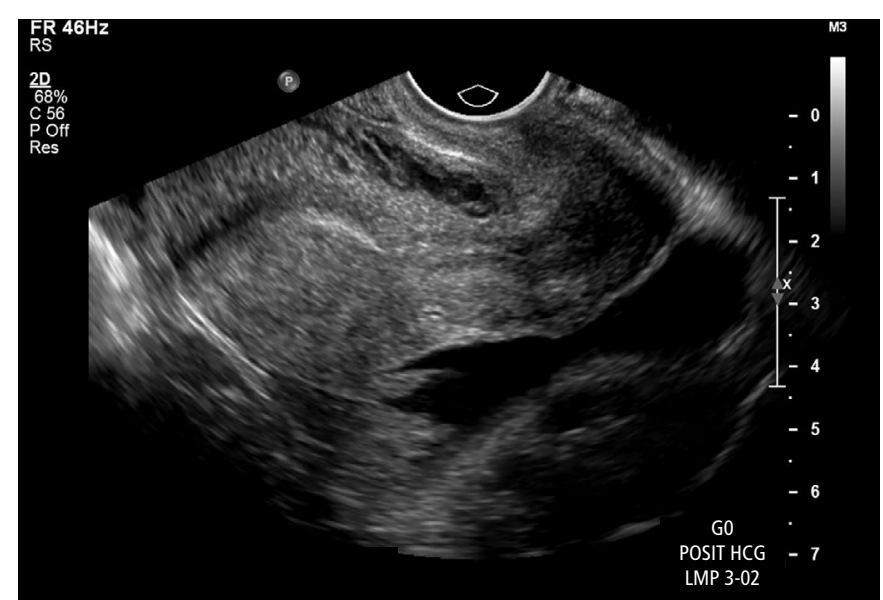

Fig. 7. Simple free fluid. In a 21-year-old woman presenting to the emergency department with pelvic pain and a positive pregnancy test, simple free fluid was found in the pelvic cul-de-sac. The patient also had an echogenic left adnexal mass (not included in this figure), which was confirmed to be an ectopic pregnancy intraoperatively.

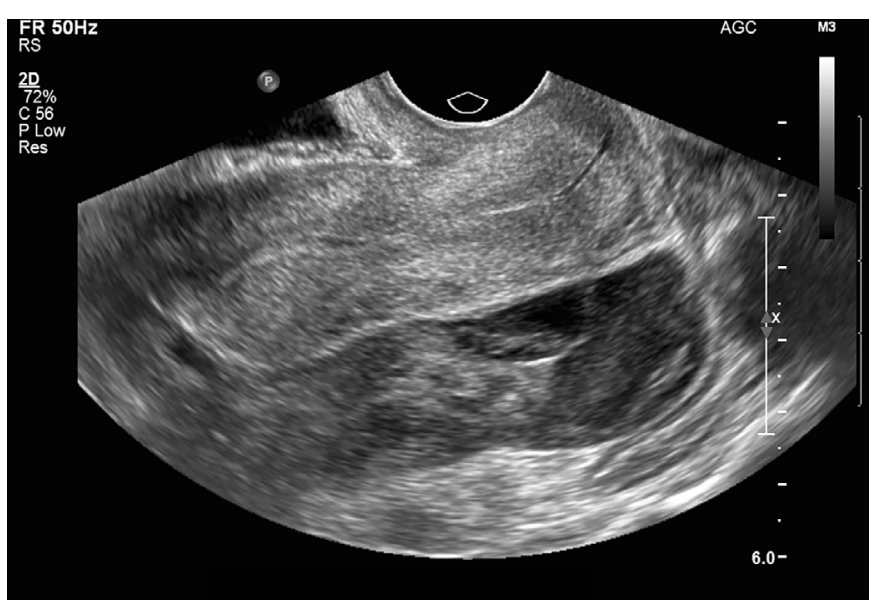

Fig. 8. Complex free fluid or hemoperitoneum. In this 22-year-old woman with a prior history of ectopic pregnancy presenting to the emergency department with pelvic pain and a positive pregnancy test, a large volume of complex free fluid with internal echogenicity was found in the pelvic cul-de-sac, most likely representing hemoperitoneum. A ruptured tubal ectopic pregnancy was confirmed intraoperatively. 


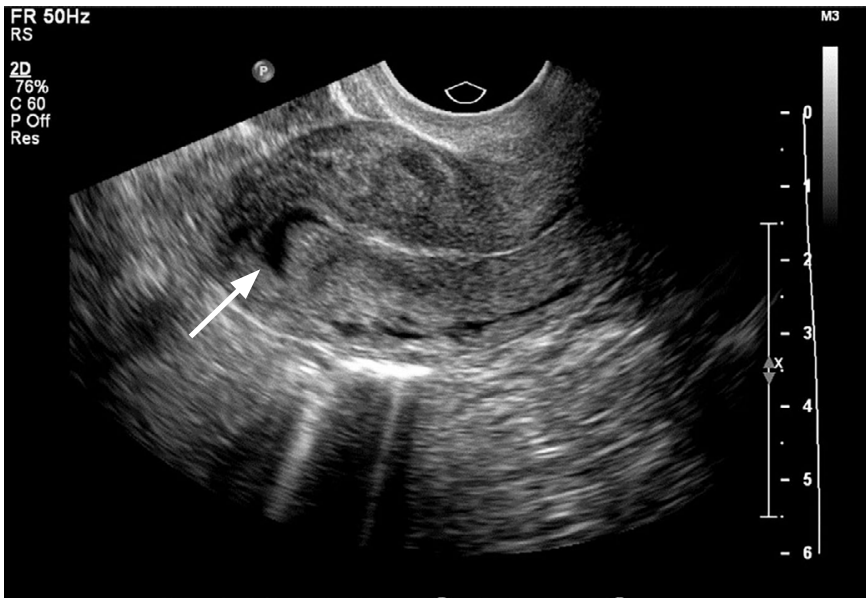

A

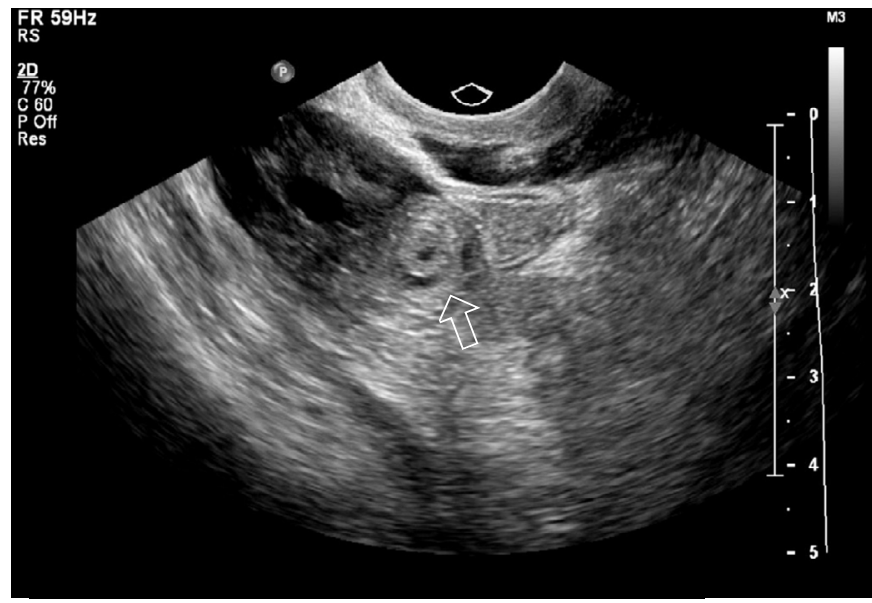

B

Fig. 9. Pseudogestational sac in an ectopic pregnancy.

A 28-year-old woman with a positive pregnancy test presented to the emergency department with right lower quadrant pain. A. A small amount of free fluid is seen within the endometrial cavity, without evidence of a yolk sac or embryo (arrow). It is irregularly-shaped and centrally located, rather than in the eccentric location often seen with a normal gestational sac. However, it should be noted that in a woman with a positive $\beta$-human chorionic gonadotropin ( $\beta$-hCG) test, any intrauterine sac-like fluid collection seen on ultrasound is highly likely to be a gestational sac. B. There is a right adnexal mass with an echogenic ring (open arrow), suspicious for ectopic pregnancy. This patient was followed clinically with serial $\beta-h C G$ testing, and she was later diagnosed with ectopic pregnancy and treated medically.

Cervical ectopic pregnancy occurs in fewer than $1 \%$ of ectopic pregnancies [11]. It is diagnosed when a gestational sac is visualized within the cervical stroma, usually in an eccentric position. The resulting cervical distention results in an hourglass-shaped uterus.

Ovarian ectopic pregnancy refers to the intraovarian implantation of a gestational sac and may account for up to $3 \%$ of ectopic pregnancies [4]. When an adnexal mass is seen, a gentle bimanual US examination should be performed to help elucidate the location of the mass relative to the ovary, as an intraovarian mass will move with the ovary with applied pressure. However, an anechoic or even complex ovarian mass is much more likely to be a corpus luteum cyst, since ovarian ectopic pregnancies are rare [5]. The diagnosis of ovarian pregnancy is often very difficult to make sonographically, and most cases are diagnosed intraoperatively [14].

Abdominal/peritoneal pregnancy is an ectopic pregnancy within the peritoneal cavity, constituting approximately $1 \%$ of ectopic pregnancies [4]. It is most often located in the pouch of Douglas (rectouterine space), but it can be anywhere within the peritoneal cavity. This can be a challenging diagnosis to make with imaging and is often made intraoperatively.

Heterotopic pregnancy is a rare condition with two concurrent pregnancies: a normal IUP and another pregnancy in an ectopic location. The most common form is a combination of an IUP with a tubal ectopic pregnancy. Heterotopic pregnancy has a significantly higher incidence among patients receiving IVF, at close to $1 \%$
[15], compared to a trivial incidence among patients who conceive naturally, at 1:30,000 [16]. Timely diagnosis and treatment may be critical not only for reducing maternal morbidity and mortality, but also for preserving the IUP.

\section{Complications of Ectopic Pregnancy}

The most common complication of ectopic pregnancy is rupture, which occurs in $15 \%-20 \%$ of ectopic pregnancies [17]. It can result in life-threatening hemorrhage and often requires emergent surgery. In a patient with a positive pregnancy test, a moderate or large hemoperitoneum without visualization of a normal IUP is highly suspicious for ruptured ectopic pregnancy (Fig. 8).

\section{US Findings after Medical Treatment}

Methotrexate has been proven to be a safe and effective treatment for ectopic pregnancy [18]. Following treatment with methotrexate, US is only indicated when there is a suspicion of tubal rupture based on persistent clinical symptoms or hemodynamic instability [5]. Sonographic findings following medical treatment can be confusing, as there is often a paradoxical enlargement of the ectopic pregnancy due to post-treatment hemorrhage and edema (Fig. 10A, B). The ectopic mass/hematoma can be visible for longer than 2 months after treatment [19]. In the context of clinical improvement and an 
appropriate decline in $\beta$-hCG levels following medical treatment, these sonographic findings usually represent the expected resolution of the ectopic pregnancy.

\section{US Mimics of Ectopic Pregnancy}

A corpus luteum (CL) may look very similar to ectopic pregnancy on
US. It is usually a round, thick-walled structure, often cystic, with varying internal echogenicity depending on presence of hemorrhage (Fig. 11A). It has marked peripheral vascular flow within the cyst wall, seen as a "ring of fire" appearance on color Doppler (Fig. 11B). The most reliable method to distinguish a $\mathrm{CL}$ from an ectopic pregnancy is to determine the location of the lesion relative to the ovary, as a CL arises from the ovary and the vast majority of ectopic

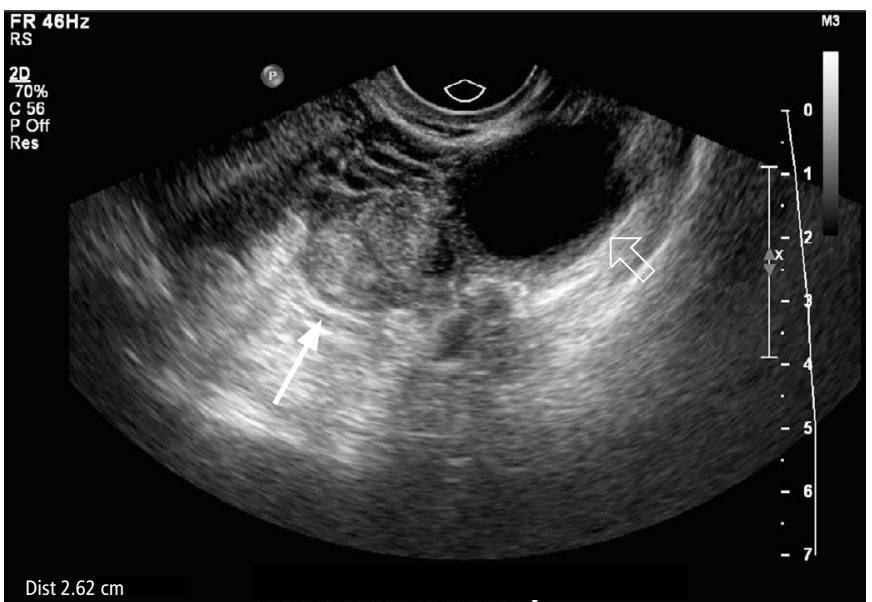

A

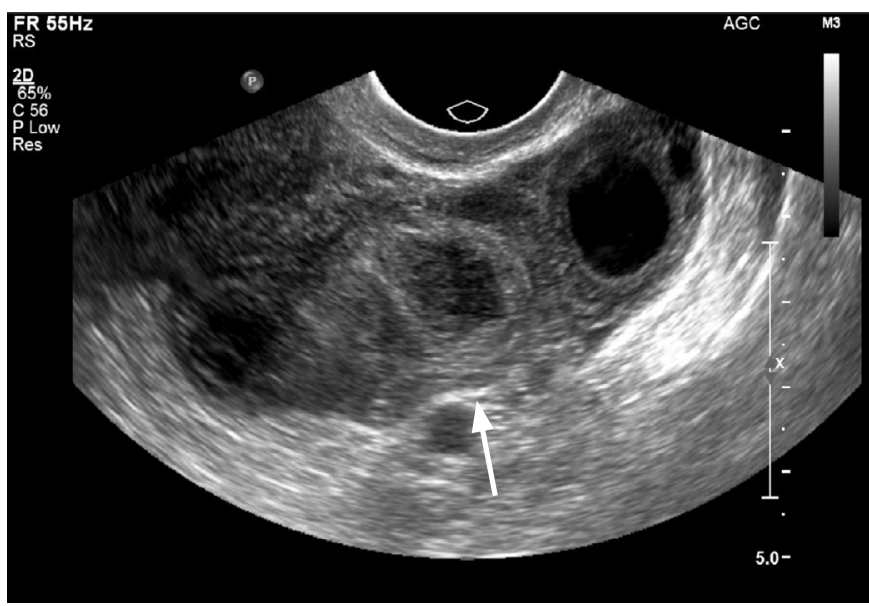

B

Fig. 10. Sonographic findings after methotrexate treatment.

This is a 31-year-old woman who has positive but declining serial $\beta$-human chorionic gonadotropin levels. A. There is a heterogeneous echogenic mass (arrow) in the left adnexa adjacent to the left ovary (open arrow). The patient was diagnosed with left tubal ectopic pregnancy and was started on medical treatment with methotrexate. B. On a follow-up pelvic ultrasound 10 days after initiation of methotrexate treatment, there is interval enlargement of the left tubal ectopic pregnancy (arrow) due to surrounding hemorrhage and edema.

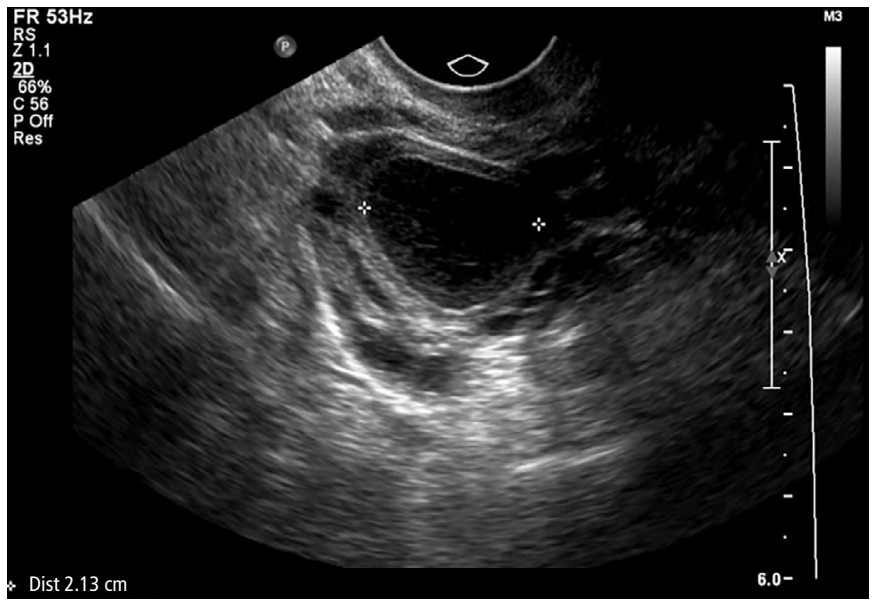

A

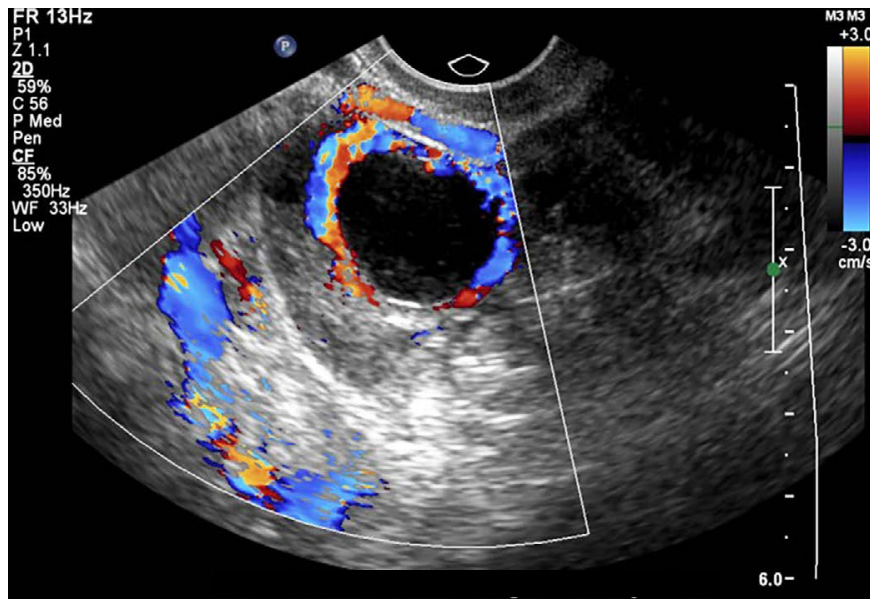

B

Fig. 11. Corpus luteum.

This figure represents a 32-year-old woman who presented to the emergency department with lower abdominal pain. A. There is an intraovarian cystic structure with echogenic ring and internal debris (indicated with cross-hair markings). This ovarian structure most likely represents a corpus luteum. B. A color Doppler ultrasonography of the same structure shows significant peripheral vascularity, which is often seen in a corpus luteum. 
pregnancies are tubal (extraovarian) [5].

An incidental adnexal mass can be mistaken for an ectopic pregnancy. Paraovarian cysts (Fig. 12) are usually simple, unilocular adnexal cysts outside of the ovary that are often found incidentally. A paraovarian cyst located adjacent to the ovary can raise concern for ectopic pregnancy, but its appearance as a simple cyst with a thin, avascular wall readily distinguishes it from an ectopic pregnancy. Dermoid cysts (Fig. 13A, B) can have variable ultrasound appearances, but are usually seen as a focal hyperechoic mass in the ovary. They can also have an echogenic-shadowing mural nodule,

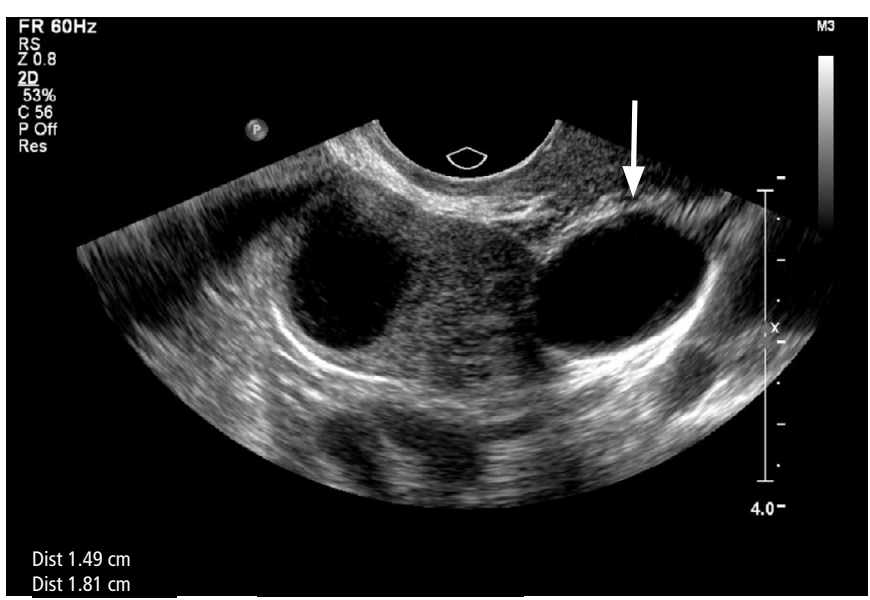

Fig. 12. Paraovarian cyst. In a 47-year-old woman, there is an anechoic cyst with a thin wall and posterior acoustic enhancement inferior to the right ovary, which is compatible with a paraovarian cyst (arrow).

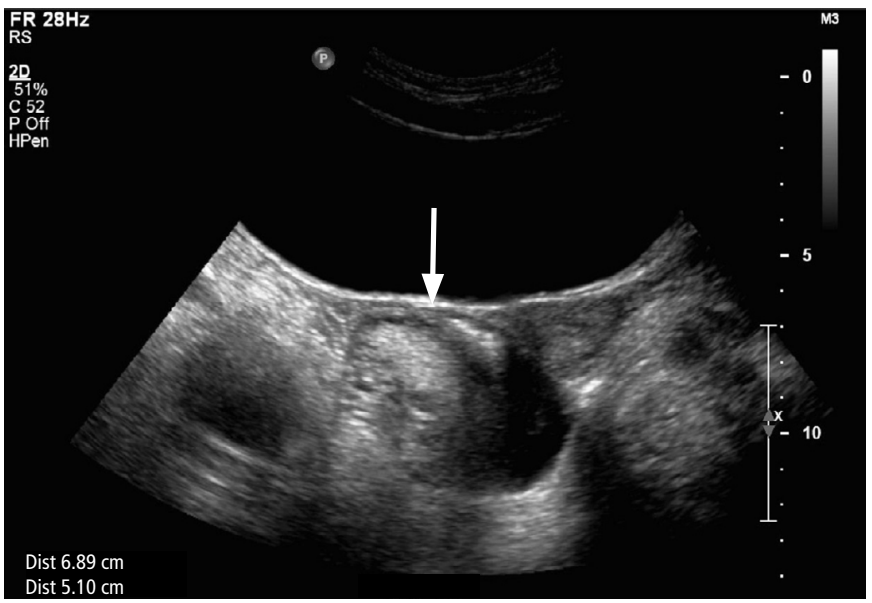

A fat-fluid level, calcifications, or dot-dash-dot appearance due to the hair. Ovarian neoplasms, although less common than paraovarian cysts or dermoid cysts, can also mimic ectopic pregnancy. If there is concern for an ovarian neoplasm based on a patient's clinical presentation and ultrasound findings, magnetic resonance imaging can be obtained for further evaluation of the mass.

Visualization of bowel loops in the pelvis (Fig. 14) can be at times misinterpreted as ectopic pregnancy, and vice versa. Bowel loops can appear as a mass-like structure with distinct walls and mural vascularity. Real-time imaging by the radiologist or Cine images provided by the technologist can be very helpful in differentiating the bowel from an ectopic pregnancy by demonstrating peristalsis in the bowel loops.

\section{Conclusion}

Ectopic pregnancy is the leading cause of pregnancy-related maternal death during the first trimester [1]. Ultrasound evaluation is an essential component of the diagnostic algorithm. In the light of the increased use of medical management and improved outcomes, the early diagnosis and timely initiation of treatment have become more critical in decreasing maternal morbidity and mortality by preventing complications and avoiding the need for surgical management. Careful clinical and imaging scrutiny of patients will result in the successful diagnosis and early management of ectopic pregnancy.

Fig. 13. Dermoid cyst.

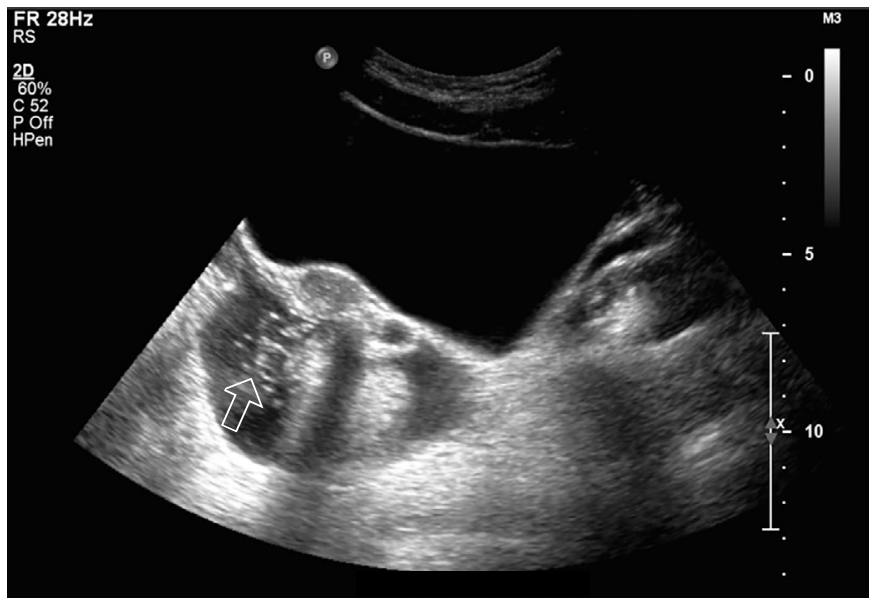

B

This is a 17-year-old girl presenting to the emergency department with pelvic pain. A. There is a complex echogenic mass with posterior acoustic shadowing (arrow). B. Multiple thin echogenic bands, also known as a dot-dash pattern (open arrow), are seen in parts of the mass, representing hair follicles. These findings are compatible with a dermoid cyst. 


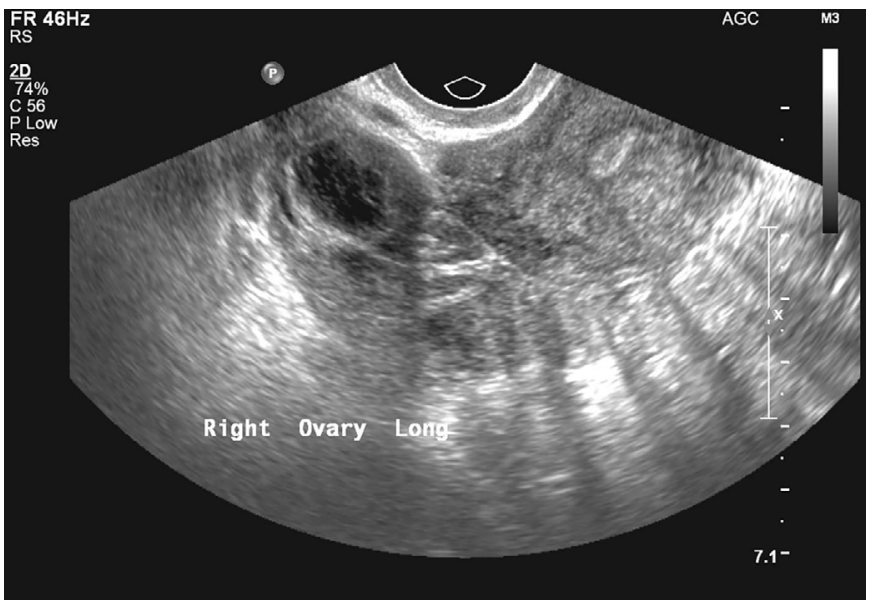

A

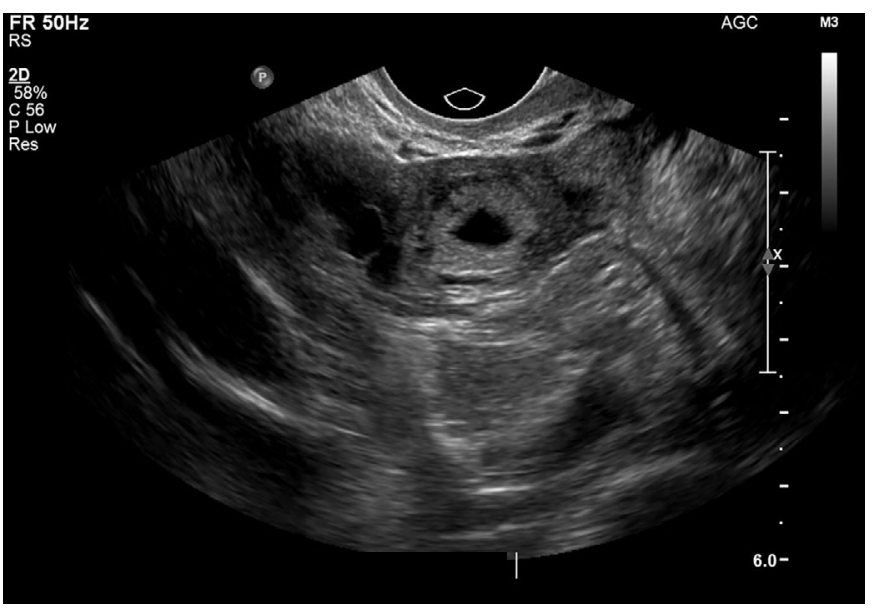

C

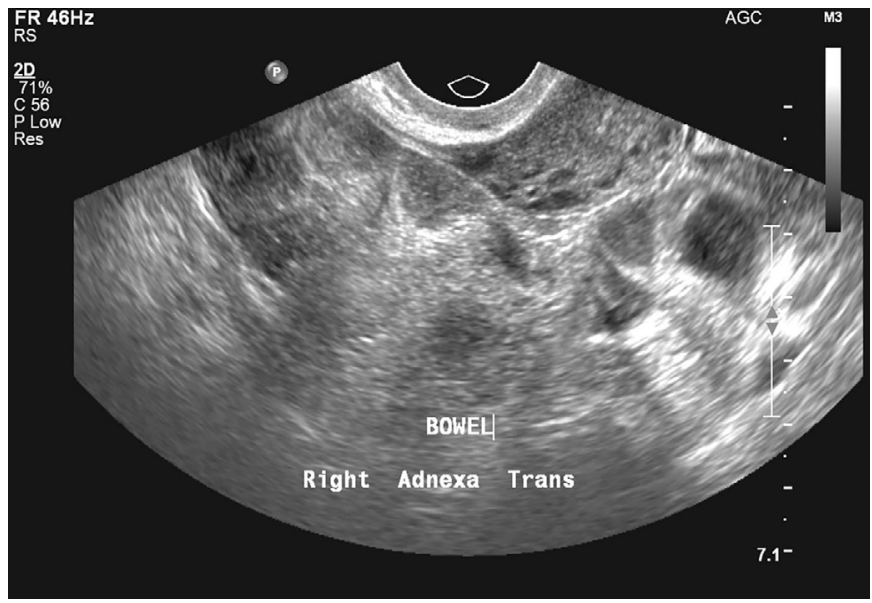

B

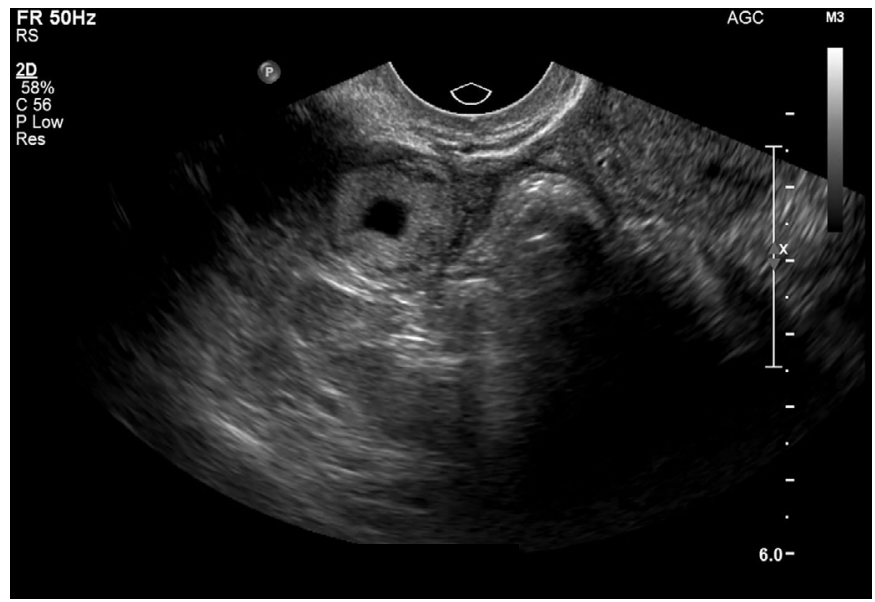

D

Fig. 14. Ectopic pregnancy misinterpreted as a bowel loop.

This figure represents a 28-year-old woman who presented to the emergency department with right lower quadrant pain and a positive pregnancy test. A. The sagittal view of the right adnexa shows a hemorrhagic cyst in the right ovary; otherwise, no definite adnexal mass can be seen. B. A transverse view of the right adnexa did not demonstrate a definitive mass in the right adnexa. At the time of the study, nonspecific echogenicity in the right adnexa was interpreted as bowel loops. C, D. The patient came back 3 days later with recurrent right lower quadrant pain. There is a mass with a thick, echogenic ring in the right adnexa, which is highly suspicious for ectopic pregnancy. The diagnosis of tubal ectopic pregnancy was confirmed intraoperatively.

ORCID: Robert Lee: http://orcid.org/0000-0003-4329-6641; Carolyn Dupuis: http:// orcid.org/0000-0003-4336-2573; Byron Chen: http://orcid.org/0000-0001-56427618; Andrew Smith: http://orcid.org/0000-0001-6947-7729; Young H. Kim: http:// orcid.org/0000-0002-0206-5663

\section{Conflict of Interest}

No potential conflict of interest relevant to this article was reported.

\section{References}

1. Centers for Disease Control and Prevention (CDC). Ectopic pregnancy: United States, 1990-1992. MMWR Morb Mortal Wkly
Rep 1995;44:46-48.

2. Murray $H$, Baakdah $H$, Bardell T, Tulandi T. Diagnosis and treatment of ectopic pregnancy. CMAJ 2005;173:905-912.

3. Goldner TE, Lawson HW, Xia Z, Atrash HK. Surveillance for ectopic pregnancy: United States, 1970-1989. MMWR CDC Surveill Summ 1993;42:73-85.

4. Bouyer J, Coste J, Fernandez H, Pouly JL, Job-Spira N. Sites of ectopic pregnancy: a 10 year population-based study of 1800 cases. Hum Reprod 2002;17:3224-3230.

5. Levine D. Ectopic pregnancy. Radiology 2007;245:385-397.

6. Mandell J. Core radiology: a visual approach to diagnostic imaging. Cambridge: Cambridge University Press, 2013. 
7. Barash JH, Buchanan EM, Hillson C. Diagnosis and management of ectopic pregnancy. Am Fam Physician 2014;90:34-40.

8. Kadar N, DeVore G, Romero R. Discriminatory hCG zone: its use in the sonographic evaluation for ectopic pregnancy. Obstet Gynecol 1981;58:156-161.

9. Dogra V, Rubens DJ. Ultrasound secrets. Philadelphia, PA: Hanley \& Belfus, 2003.

10. Kaakaji Y, Nghiem HV, Nodell C, Winter TC. Sonography of obstetric and gynecologic emergencies: Part I, Obstetric emergencies. AJR Am J Roentgenol 2000;174:641-649.

11. Lin EP, Bhatt S, Dogra VS. Diagnostic clues to ectopic pregnancy. Radiographics 2008;28:1661-1671.

12. Ackerman TE, Levi CS, Dashefsky SM, Holt SC, Lindsay DJ. Interstitial line: sonographic finding in interstitial (cornual) ectopic pregnancy. Radiology 1993;189:83-87.

13. Jurkovic D, Hillaby K, Woelfer B, Lawrence A, Salim R, Elson CJ. Firsttrimester diagnosis and management of pregnancies implanted into the lower uterine segment Cesarean section scar. Ultrasound Obstet Gynecol 2003;21:220-227.
14. Raziel A, Schachter M, Mordechai E, Friedler S, Panski M, RonEl R. Ovarian pregnancy-a 12-year experience of 19 cases in one institution. Eur J Obstet Gynecol Reprod Biol 2004;114:92-96.

15. Luo X, Lim CE, Huang C, Wu J, Wong WS, Cheng NC. Heterotopic pregnancy following in vitro fertilization and embryo transfer: 12 cases report. Arch Gynecol Obstet 2009;280:325-329.

16. Govindarajan MJ, Rajan R. Heterotopic pregnancy in natural conception. J Hum Reprod Sci 2008;1:37-38.

17. Job-Spira N, Fernandez H, Bouyer J, Pouly JL, Germain E, Coste J. Ruptured tubal ectopic pregnancy: risk factors and reproductive outcome: results of a population-based study in France. Am J Obstet Gynecol 1999;180:938-944.

18. Barnhart KT, Gosman G, Ashby R, Sammel M. The medical management of ectopic pregnancy: a meta-analysis comparing "single dose" and "multidose" regimens. Obstet Gynecol 2003;101:778-784.

19. Gamzu R, Almog B, Levin Y, Pauzner D, Lessing JB, Jaffa A, et al. The ultrasonographic appearance of tubal pregnancy in patients treated with methotrexate. Hum Reprod 2002;17:2585-2587. 